\title{
Epidemiological and Pathological Correlates of Postoperative Mortality of Patients with Ovarian Cancer
}

\author{
Refaat A Hegazi', Khaled Abdel Wahab², Waleed El Nahas ${ }^{2}$, Mahmoud Mosbah², Basel Refky² and Mohamed A Hegazy ${ }^{2 *}$ \\ ${ }^{1}$ Faculty of Medicine, Department of Community Medicine, Mansoura University, Egypt \\ ${ }^{2}$ Surgical Oncology Center, Mansoura University, Egypt
}

\section{Abstract}

Background: Epithelial ovarian cancer is the most lethal gynecologic malignancy and is the fifth most common cause of cancer-related death among women. The current study was designed to investigate the epidemiological and pathological correlates of postoperative mortality of ovarian cancer at a tertiary care center in Mansoura, Egypt.

Methods: An epidemiological analysis of prospectively collected data of ninety five primary ovarian cancer cases referred to the Surgical Oncology Center at Mansoura University Hospitals, Mansoura, Egypt. The association of the epidemiological, pathological data and the serum levels of the tumor marker CA-125 and 6-months and 1-year mortality was statistically tested.

Results: A total of 95 ovarian cancer cases were included in the current study. The mean age of patients was 52.18 ranging from 14 to 98 years, and the median age was 53 years. Pathological examination showed that serous cancers were the most common type detected in $46(48 \%)$, followed by adenocarcinoma in $24(25 \%)$ and then mucinous in $9(9 \%)$ patients. Cases presented at late stages of the ovarian cancer disease with $51(55 \%)$ cases presenting with stage III and IV. The majority of cases $(n=83,88.1 \%)$ received neo adjuvant chemotherapy. The average serum CA125 level was $325.38 \mathrm{U} / \mathrm{ml}$ with $50 \%$ of cases had readings equal to or more than $233.15 \mathrm{U} / \mathrm{ml}$. The mortality rate at 6 months was $4 \%$ and at 1 -year was $17 \%$. Age was positively correlated with 6 months mortality $(p<0.05)$. Ninety percent of deaths at one year occurred in patients more than 45 years of age and $50 \%$ of deaths occurred in patients more than 60 years of age.

Conclusion: In a tertiary care surgical oncology center in Mansoura, Egypt, patients with ovarian cancer are more commonly to present with later stage at the postmenopausal age and the age at diagnosis is positively associated with increased postoperative mortality.

Keywords: Ovarian cancer; Mortality; Epidemiology; Pathology

\section{Introduction}

Epithelial ovarian cancer (EOC) is the most lethal gynecologic malignancy and is the fifth most common cause of cancer-related death among women [1].

In Egypt, a regional registry, the Gharbia Population Based Cancer Registry (GPBCR), showed data of 225 ovarian cancer cases during a three years period (2000-2002). The registry showed that ovarian cancer represented $2.2 \%$ of all incident cancers and accounted for $4.4 \%$ of all newly diagnosed female cancers [2]. Another important regional registry in Egypt is the Aswan regional registry, in which thirty-five cases of ovarian cancer were registered in 2008, representing $5.6 \%$ of all female cancers cases [3].

About $70 \%$ of newly diagnosed ovarian cancer patients will have advanced disease and often not totally resectable due to the lack of symptoms in the early stages of ovarian cancer. The prognosis of patients with ovarian cancer is poor, with a 5 year survival of about $35 \%$ [4]. Owing to improvements in diagnosis, surgery, and chemotherapy, during the past 30 years, survival has increased [5].

The identification of prognostic factors in studies of ovarian cancer may be useful for a variety of reasons. It would increase the understanding of the natural history of the disease, provide clinicians with guidelines for decisions on treatment strategies, and adjust for imbalances in comparing therapeutic regimens. Numerous studies have attempted to identify the clinical and pathologic correlates of the disease outcomes and the factors that could provide prognostic information for individual patient survival [6-8].

The current study was designed to describe the epidemiological and pathological correlates of ovarian carcinoma cases admitted for surgical treatment at the surgical oncology unit in Mansoura oncology Center, Mansoura, Egypt.

\section{Materials and Methods}

\section{Patients}

This is an epidemiological analysis of prospectively collected data of ninety five patients with ovarian cancer referred to the surgical oncology unit of Mansoura University Hospital, Mansoura, Egypt between the period of June 2008 and June 2012. The study was approved by the local ethical committee of Mansoura University Faculty of Medicine and all patients provided written informed consent prior to participation.

\section{Staging of ovarian cancer}

Ovarian cancer staging by the FIGO staging system uses information obtained after surgery, which can include a total abdominal hysterectomy, bilateral salpingo-oophorectomy, infracolic omentectomy, and peritoneal washings for cytopathology. The AJCC stage is the same as the FIGO stage. The AJCC staging system describes the extent of the primary Tumor $(\mathrm{T})$, the absence or presence of

${ }^{*}$ Corresponding author: Mohamed A Hegazy, Surgical Oncology Center, Mansoura University, Egypt, E-mail: mhegazy68@yahoo.com

Received December 12, 2012; Accepted January 25, 2013; Published February 03, 2013

Citation: Hegazi RA, Wahab KA, Nahas WE, Mosbah M, Refky B, et al. (2013) Epidemiological and Pathological Correlates of Postoperative Mortality of Patients with Ovarian Cancer. Surgery Curr Res 3: 126. doi:10.4172/2161-1076.1000126

Copyright: (c) 2013 Hegazi RA, et al. This is an open-access article distributed under the terms of the Creative Commons Attribution License, which permits unrestricted use, distribution, and reproduction in any medium, provided the original author and source are credited. 
metastasis to nearby lymph Nodes $(\mathrm{N})$, and the absence or presence of distant Metastasis (M) [9].

\section{Serum CA-125 measurement}

Serum CA-125 levels were measured using an immune assay performed by IMMULITE 1000 systems (Siemens, Los Angeles, CA, USA).

\section{Operative Strategy}

Patients were subjected to physical examination, serum level of CA 125 measurement, radiological studies, and histopathologic confirmation of ovarian carcinoma. All patients were admitted for surgical exploration at the Surgical Oncology Unit, Mansoura University Hospitals. The purpose of this exploration was to evaluate tumor resectability; to perform primary debulking surgery when optimal cytoreduction seemed feasible and to treat primary unresectable tumors with neoadjuvant chemotherapy. Optimal debulking has been variously defined, however we adopted the Gynecologic Oncology Group definition which defines it as leaving residual disease of less than $1 \mathrm{~cm}[10]$.

This strategy was explained to the patients and informed consents were obtained. Surgical exploration was usually done laparoscopically unless it was contraindicated, when laparotomy was done. Neoadjuvant chemotherapy was given when we considered that optimal cytoreduction was not feasible with the standard surgery, defined as 1) total abdominal hysterectomy with bilateral salpingo oophorectomy, 2) appendectomy, 3) total infragastric omentectomy, 4) peritonectomy limited to the pelvis, paracolic gutters, anterolateral diaphragmatic area, and 5) pelvic, common iliac, and infrarenal paraaortic lymphadenectomy. Conversely primary debulking surgery was performed when we considered that optimal cytoreduction could be achieved by the standard surgery ( 32 patients). However, in a few cases non-standard surgery, meaning a single organ resection (e.g., small intestine, colon, spleen) in the way to achieve an optimal cytoreduction was adopted.

\section{Statistical Analysis}

Data were represented as the mean (median) and standard error of the mean. Differences among groups for quantitative variables were evaluated using the Kruskal-Wallis test and for categorical data using the $X^{2}$ or Fisher exact test. All analyses were performed utilizing the Statistical Packages for Social Sciences (SPSS, version 20, Chicago, IL). For statistical analysis, testing was two-sided and $\mathrm{p}<0.05$ was considered statistically significant.

\section{Results}

We identified 103 patients with documented operative diagnosis of ovarian cancer. We then excluded 8 patients with pathological diagnoses of borderline and Krukenberg tumors. Therefore, the current analysis includes 95 patients with primary ovarian cancer who were admitted to the surgical oncology unit of the Mansoura Oncology center and underwent different types of surgeries.

\section{Age of patients at diagnosis}

The mean age of patients in this study was 52.18 ranging from 14 to 98 years, and the median age was 53 years. The age distribution showed an age peak that lied between 45 and 62 years of age (Figure 1). Seven cases $(7 \%)$ were at the age of 30 years or less.

\section{Menstrual history}

Data available for the menstrual history of the patients in the

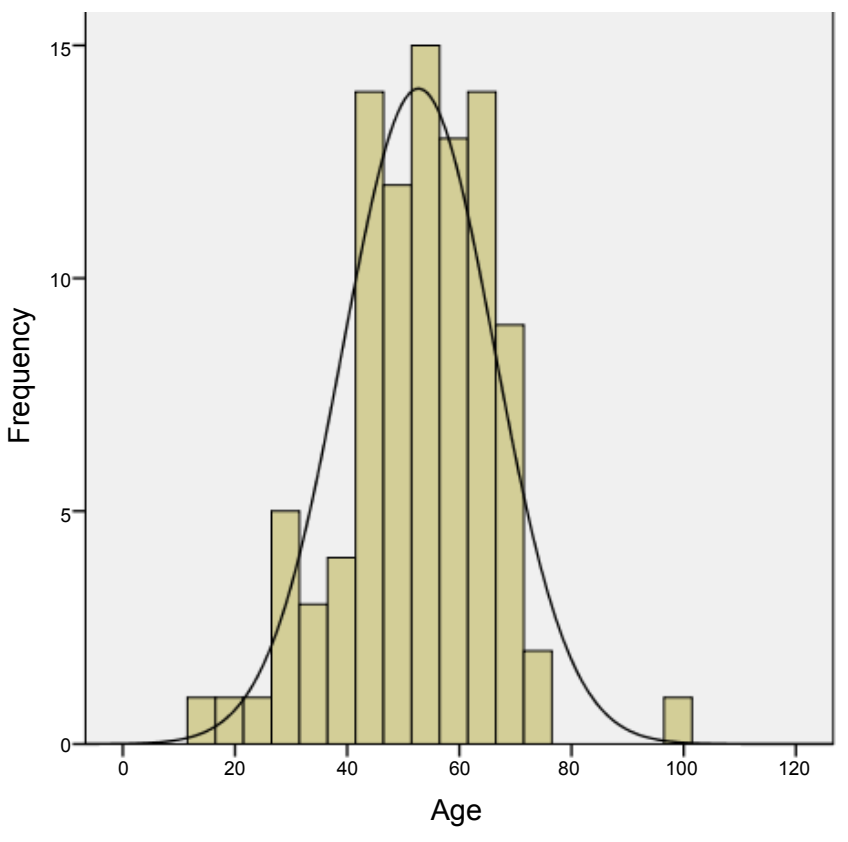

Figure 1: Age distribution of the patients with primary ovarian cancer $(n=95)$.

current cohort showed that the mean age of menarche was 13.13 and the median age is 13 . Moreover, $72(76 \%)$ ovarian cancer patients in the current study series were postmenopausal.

\section{Comorbid medical disease}

The majority of the patients did not have significant comorbidity with only $10(10 \%)$ patients have a diagnosis of hypertension and 3 (3\%) have a diagnosis of type $2 \mathrm{DM}$.

\section{Pathological types}

Pathological examination showed that serous cancers were detected in 46 (48\%) cases, followed by adenocarcinoma in 24 (25\%) of cases and the mucinous type in $9(9 \%)$. Undifferentiated and granulosa cell tumors counted next with equal percent of cases $(n=4,4.6 \%)$ while endometrioid tumors were found in $3(3.4 \%)$ cases. Other types were found in our series as mullerian tumors $(n=3)$, germ cell $(n=1)$ and sarcomas $(n=1)$ with smaller percentiles $(3,1$ and $1 \%$, respectively).

\section{Bilateral disease}

Unilateral tumors were found more commonly than bilateral tumors. Unilateral tumors were found in $55(65 \%)$ cases while patients with bilateral tumors represented only 30 (35\%) cases.

\section{Serum levels of the tumor biomarker CA-125}

The serum levels of the tumor biomarker, CA-125, were available for 67 patients. The average level is $325.38 \mathrm{U} / \mathrm{ml}$ with $50 \%$ of cases had readings below or equal to $233.15 \mathrm{U} / \mathrm{ml}$. We studied the serum levels of the CA-125 biomarker in association with the pathological type of the tumor (Figure 2). Patients with serous tumors had statistically significantly greater values of CA-125 than patients with mucinous tumors $(374.59 \pm 332.68$ versus $51.80 \pm 54.59 \mathrm{U} / \mathrm{ml}, \mathrm{p}=0.04)$. Moreover, the granulosa cell tumors were associated with the least serum levels of CA-125 $(21.31 \pm 16.55)$ and generally occurred in younger patients (average age 42 years). 


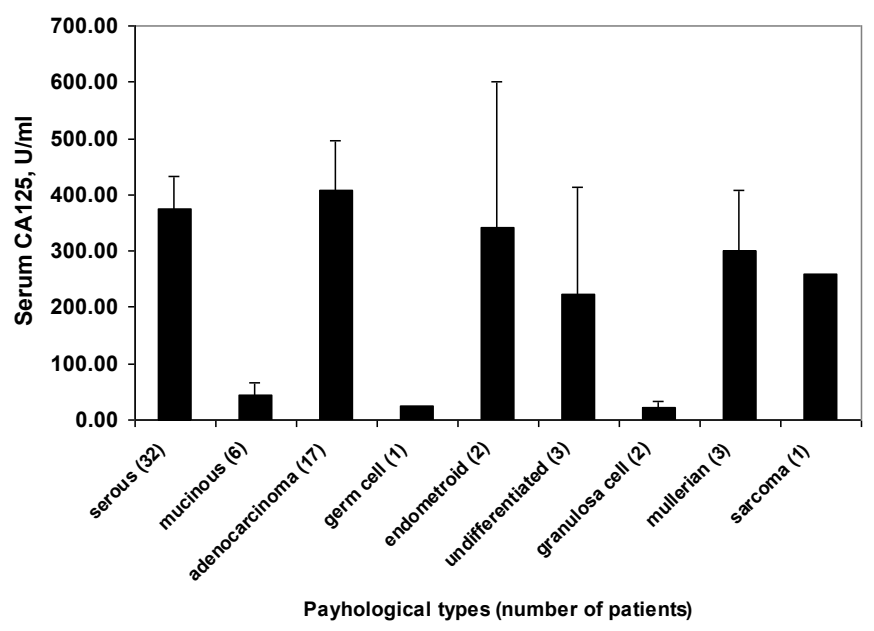

Figure 2: Average serum CA-125 levels by the pathological type in patients with primary ovarian cancer $(n=67)$.

\section{Staging of the tumor and the use of neo adjuvant chemo therapy}

Cases presented at late stages of the ovarian cancer disease with $51(55 \%)$ cases presenting with stage III and IV. The majority of cases $(n=83,88.1 \%)$ in the current cohort received neo adjuvant chemotherapy. This may be attributed to the large proportion of cases first diagnosed at later stages of the disease. The age distribution of different stages is illustrated in table 1 . As shown in the table, there is a trend of an older age with higher stage, but this association was not statistically significant. Specifically, the median age of patients with stage 1 is 48 years while the median age of patients with stage IV is 59 years. However, the statistical comparison of the means among the four stages was not significant $(p=0.41)$. Illustrated in the table as well are the CA-125 levels of patients at the different stages of ovarian cancer. Patients with stage I had the lowest levels of CA-125 while patients with stage III had the highest levels.

\section{Correlates of postoperative mortality}

The mortality rate at 6 months was $4 \%$ while the 1 -year mortality rate is $17 \%$. We investigated the association between mortality and the epidemiological and pathological variables studied in this cohort. Interestingly, age was positively correlated with 6 months mortality. Although the same trend was observed of the association of age and 1 -year mortality ( $90 \%$ of deaths at 1 year occurred in patients more than 45 years of age), this association was not statistically significant (Table 2). In this cohort of ninety five patients with ovarian cancer, no significant association was detected between mortality and any of the other variables.

\section{Discussion}

The current study reports the epidemiological and pathological characteristics of patients with ovarian cancer and their association with the 6-months and 1-year postoperative mortality of patients referred to a tertiary care center at Mansoura, Egypt. We show that only the age at diagnosis was positively associated with 6-months postoperative mortality.

The average age of ovarian cancer patients in the current study was 53 years old, which is slightly different from other studies. Interestingly, seven cases were 30 years old or less. Of these cases, three had serous carcinoma, two had mucinous, one granulosa cell and one germ cell tumors. At 1-year, all four cases, for whom survival data were available, survived. DiSilvestro et al. [11] observed that the mean age in the study that included one hundred and thirty seven cases was 58 years old while Mostafa et al. [12] stated that the mean age of one hundred and sixteen Egyptian patients was 46 years. The median age at menarche in the current study was 13 years old. The relationship between the age at menarche and ovarian cancer is unclear. Several studies have reported an increased risk of ovarian cancer with early menarche [13-15], while others have shown no significant association [16,17]. We could not find any association of the age of menarche with either the stage of the tumor or with mortality (data not shown).

In the current report, patients with ovarian cancer commonly presented for surgical intervention at a later stage with $55 \%$ of patients presenting with stage III and IV. This was consistent with previous findings. For instance, Hornung et al. [18], stated that nearly more than half of cases presented with either stage III or IV ( $46 \%$ and $12 \%$, respectively). However, Mostafa et al. [12] showed that approximately $80 \%$ of ovarian cancer cases presented at stage III and IV $(41.3 \%$ and $37.9 \%$, respectively).

Serous adenocarcinoma was the most common diagnostic tumor pathology found in 39\% of ovarian cancer cases in the current study. This is in agreement with the study by Beard et al. [19] of one hundred and twenty nine ovarian cancer cases. They found that serous adenocarcinomas constituted $43 \%$ of their cases while Mostafa et al. [12] reported that serous adenocarcinoma was found in $58 \%$ of cases in their study.

CA-125 is the biomarker most frequently used for the detection of ovarian cancer being elevated in approximately $90 \%$ of patients with advanced ovarian cancer. In the current study, the median serum levels of the CA-125 in patients with stage I patients is $62 \mathrm{U} / \mathrm{ml}$ was significantly lower than the levels in patients with stage III-IV (294.5 U/ $\mathrm{ml})$. These results are consistent with previous literature. Yedema et al. [20] noted that in patients with stage I-II ovarian cancer, pre-operative CA 125 serum levels were significantly lower compared to the CA 125 serum levels in patients with stage III-IV (median: $135 \mathrm{U} / \mathrm{ml}$ vs. 2000 $\mathrm{U} / \mathrm{ml}$, respectively).

Of note, other correlates of survival were not included in the current study. For instance, the presence of a BRCA1 or BRCA2 mutation was associated with a better prognosis in a recent study [21]. Exploring the association of postoperative outcome with these biomarkers is warranted in future studies.

\begin{tabular}{|l|c|c|}
\hline Tumor Stage (n) & $\begin{array}{c}\text { Age } \\
\text { Mean, } \\
\text { (Median, SEM) }\end{array}$ & $\begin{array}{c}\text { CA-125 } \\
\text { Mean } \\
\text { (Median, SEM) }\end{array}$ \\
\hline I (20) & $49.1(47.5,3.6)$ & $213.4(62,55.6)$ \\
II (12) & $48.1(48.5,3.9)$ & $327.9(226,94.3)$ \\
III (34) & $52.3(53.0,1.8)$ & $400.8(348.5,61.4)$ \\
IV (6) & $57.0(59.5,5.0)$ & $266.4(196.9,108.3)$ \\
\hline
\end{tabular}

Table 1: The age and CA-125 serum levels of patients with primary ovarian cancer by tumor stages.

\begin{tabular}{|l|c|c|c|c|c|}
\hline \multirow{2}{*}{ Number (\%) } & \multicolumn{3}{|c|}{ Age Groups (years) } & \multirow{2}{*}{ P-value } \\
\cline { 3 - 6 } & & $<45$ & $45-61$ & $>61$ & \\
\hline 6-Months & Yes & $22(28)$ & $35(45)$ & $21(27)$ & \multirow{2}{*}{0.02} \\
\cline { 1 - 5 } Survival & No & $0(0)$ & $0(0)$ & $3(100)$ & \\
\hline \multirow{2}{*}{ 1-Year Survival } & Yes & $15(31)$ & $21(43)$ & $13(27)$ & \multirow{2}{*}{0.17} \\
\cline { 3 - 5 } & No & $1(10)$ & $4(40)$ & $5(50)$ & \\
\hline
\end{tabular}

Table 2: Postoperative survival at 6 months and 1 year by age groups. 
The majority of cases $(88.1 \%)$ in the current cohort received neo adjuvant chemotherapy. This may be attributed to the large proportion of cases first diagnosed at later stages of the disease. We previously reported our prospective study including 59 patients with stage III or IV epithelial ovarian carcinomas who after mini-laparotomy and thorough assessment of the possibility of attaining optimal cytoreduction, 32 patients underwent primary cytoreductive surgery, and 27 patients were closed and received neoadjuvant chemotherapy. The study showed that the primary cytoreductive surgery is still the gold standard for the treatment of ovarian carcinoma. The results of this study directed our operative and neoadjuvant chemotherapy protocol at Mansoura Surgical Oncology center for the management of ovarian cancer. Neoadjuvant chemotherapy for advanced unresectable ovarian carcinoma leads to the selection of patients sensitive to chemotherapy, in whom secondary cytoreductive surgery can be achieved in a less aggressive manner and prevents unwarranted surgery in patients with a very poor prognosis either because of progressive disease or because of primary chemoresistance [22]. This is consistent with Bristow et al. who reported in a meta-analysis that two-thirds of patients are not candidates for optimal primary cytoreduction [23]. However, others have achieved better results using more extended surgery [24]. Whether suboptimal cytoreduction is the consequence of a more aggressive tumor or a less aggressive surgical approach is still controversial. This significant subset of patients undergoing suboptimal debulking will not derive any benefit from this procedure, but will suffer the morbidity of such an intervention. The alternative to primary surgery in patients with an unresectable tumor or poor performance status is neoadjuvant chemotherapy. Retrospective studies have shown comparable survival results in patients undergoing neoadjuvant chemotherapy, and reported significantly higher rates of subsequent optimal surgical cytoreduction and less postoperative morbidity $[25,26]$.

The mortality rate at 6 months was $4 \%$ while the 1 -year mortality rate is $17 \%$. We investigated the association between mortality and the epidemiological and pathological variables studied in this cohort. Interestingly, age was positively correlated with 6 months mortality. Although the same trend was observed of the association of age and 1 -year mortality ( $90 \%$ of deaths at 1 year occurred in patients more than 45 years of age), this association was not statistically significant (Table 2). Moreover, all three deaths at 6-months were all stage III ovarian cancer. No significant association was detected between mortality and any of the other studied epidemiological and reproductive variables. This finding is in agreement with Lang et al. [17] in their study of the predictors of survival of a cohort of 635 epithelial ovarian cancer cases derived from a nationwide population-based case-control study conducted in Sweden. The study showed that the tumor characteristics significantly influenced the risk of death and that no association was detected between survival and any of the studied pre-diagnosis reproductive, anthropometric, lifestyle or family history factors.

The current study has some methodological limitations notably the small sample size and the incomplete accrual of some variables. However, it also has many areas of strength. Importantly, this is the first cohort to study the epidemiological and pathological correlates of postoperative survival of patients with ovarian cancer living in this area of northern Egypt. The inclusion of patient characteristics, tumor pathological variables and CA-125 levels and testing their correlation with postoperative mortality also adds to the strength of the current study. In the future, we plan to continue following up these variables and to test these associations in a larger cohort.

In summary, in a cohort of 95 patients with ovarian cancer at a tertiary care center in Mansoura, Egypt, patients present with at a late stage of the disease and age correlates positively with postoperative mortality.

\section{Acknowledgements}

All authors participated in the design of the study and drafting the manuscript. RAH conducted the statistical analysis. KAW, BR, AD and MAH collected the clinical data. This work was supported by the Mansoura Surgical Oncology Department Mansoura University Hospital. The authors thank Doaa Samy, MD and Nermin el Lakany, MD for their assistance with the data collection.

\section{References}

1. Jemal A, Tiwari RC, Murray T, Ghafoor A, Samuels A, et al. (2004) Cancer statistics, 2004. CA Cancer J Clin 54: 8-29.

2. Ibrahim Abd el-bar (2007). Cancer in Egypt, Gharbiah. Triennial report of 20002002, Gharbiah population based cancer registry.

3. Egypt National Cancer Registry, Aswan Profile (2008) Ministry of Communication and Information Technology.

4. Runnebaum IB, Stickeler E (2001) Epidemiological and molecular aspects of ovarian cancer risk. J Cancer Res Clin Oncol 127: 73-79.

5. Harries M, Gore M (2002) Part I: chemotherapy for epithelial ovarian cancertreatment at first diagnosis. Lancet Oncol 3: 529-536.

6. Chi DS, Liao JB, Leon LF, Venkatraman ES, Hensley ML, et al. (2001) Identification of prognostic factors in advanced epithelial ovarian carcinoma. Gynecol Oncol 82: 532-537.

7. Ozols RF, Garvin AJ, Costa J, Simon RM, Young RC (1980) Advanced ovarian cancer: correlation of histologic grade with response to therapy and survival. Cancer 45: 572-581.

8. Neijt JP, ten Bokkel Huinink WW, van der Burg ME, van Oosterom AT, Willemse $\mathrm{PH}$, et al. (1991) Long-term survival in ovarian cancer. Mature data from The Netherlands Joint Study Group for Ovarian Cancer. Eur J Cancer 27 1367-1372.

9. Edge SB, Byrd DR, Compton CC, Fritz AG, Greene FL et al. (2010) Ovary and primary peritoneal carcinoma. In: AJCC Cancer Staging Manual. (7thedn), New York, NY: Springer-Verlag.

10. Gallo A, Frigerio L (2003) Neoadjuvant chemotherapy and surgica considerations in ovarian cancer. Curr Opin Obstet Gynecol 15: 25-31.

11. DiSilvestro P, Peipert JF, Hogan JW, Granai CO (1997) Prognostic value of clinical variables in ovarian cancer. J Clin Epidemiol 50: 501-505.

12. Mostafa MF, El-etrebya N, Awad N (2012) Retrospective analysis evaluating ovarian cancer cases presented at the clinical oncology department, Alexandria University. Alex J Med 48: 353-360.

13. Chiaffarino F, Pelucchi C, Parazzini F, Negri E, Franceschi S, et al. (2001) Reproductive and hormonal factors and ovarian cancer. Ann Oncol 12: 337341.

14. Shu XO, Brinton LA, Gao YT, Yuan JM (1989) Population-based case-contro study of ovarian cancer in Shanghai. Cancer Res 49: 3670-3674.

15. Jordan SJ, Webb PM, Green AC (2005) Height, age at menarche, and risk of epithelial ovarian cancer. Cancer Epidemiol Biomarkers Prev 14: 2045-2048.

16. Titus-Ernstoff L, Perez K, Cramer DW, Harlow BL, Baron JA, et al. (2001) Menstrual and reproductive factors in relation to ovarian cancer risk. $\mathrm{Br} \mathrm{J}$ Cancer 84: 714-721.

17. Chen Y, Wu PC, Lang JH, Ge WJ, Hartge P, et al. (1992) Risk factors for epithelial ovarian cancer in Beijing, China. Int J Epidemiol 21: 23-29.

18. Hornung R, Urs E, Serenella E, Edward W, Ursula S, et al. (2004) Analysis of potential prognostic factors in 111 patients with ovarian cancer. Cancer Lett 206: $97-106$

19. Beard CM, Hartmann LC, Atkinson EJ, O'Brien PC, Malkasian GD, et al. (2000) The epidemiology of ovarian cancer: a population-based study in Olmsted County, Minnesota, 1935-1991. Ann Epidemiol 10: 14-23.

20. Yedema CA, Kenemans P, Voorhorst F, Bon G, Schijf C, et al. (1993) CA 125 half-life in ovarian cancer: a multivariate survival analysis. $\mathrm{Br} \mathrm{J}$ Cancer 67 1361-1367.

21. McLaughlin JR, Rosen B, Moody J, Pal T, Fan I, et al. (2013) Long-Term Ovarian Cancer Survival Associated With Mutation in BRCA1 or BRCA2. J Nat Cancer Inst 105: 141-148. 
Citation: Hegazi RA, Wahab KA, Nahas WE, Mosbah M, Refky B, et al. (2013) Epidemiological and Pathological Correlates of Postoperative Mortality of Patients with Ovarian Cancer. Surgery Curr Res 3: 126. doi:10.4172/2161-1076.1000126

22. Hegazy MA, Hegazi RA, Elshafei MA, Setit AE, Elshamy MR, et al. (2005) Neoadjuvant chemotherapy versus primary surgery in advanced ovarian carcinoma. World J Surg Oncol 3: 57.

23. Bristow RE, Tomacruz RS, Armstrong DK, Trimble EL, Montz FJ (2002) Survival effect of maximal cytoreductive surgery for advanced ovarian carcinoma during the platinum era: a meta-analysis. J Clin Oncol 20: 1248-1259.

24. Eisenkop SM, Friedman RL, Wang HJ (1998) Complete cytoreductive surgery is feasible and maximizes survival in patients with advanced epithelial ovarian cancer: a prospective study. Gynecol Oncol 69: 103-108.

25. Giede KC, Kieser K, Dodge J, Rosen B (2005) Who should operate on patients with ovarian cancer? An evidence-based review. Gynecol Oncol 99: 447-461.

26. Crawford SC, Vasey PA, Paul J, Hay A, Davis JA, et al. (2005) Does aggressive surgery only benefit patients with less advanced ovarian cancer? Results from an international comparison within the SCOTROC-1 Trial. J Clin Oncol 23: 8802-8811. 